\title{
Personalised CFTR pharmacotherapeutic response testing and therapy of cystic fibrosis
}

\author{
To the Editor:
}

Cystic fibrosis (CF) is a fatal, multisystem, genetic disorder caused by mutations in the cystic fibrosis transmembrane conductance regulator (CFTR), a chloride transporter critical to luminal fluid homeostasis at respiratory airway and other ductal epithelial surfaces. Therapeutic options for some CF patients were transformed by the approval of ivacaftor and lumacaftor/ivacaftor, which are indicated, respectively, for patients heterozygous for a subset of rare class III mutations [1] or homozygous for the common class II mutation Phe508del [2]. Notwithstanding, more than $40 \%$ of CF patients do not meet the CFTR mutation-specific inclusion criteria and are excluded from these therapies and, while it is impractical to conduct a clinical trial for each new pharmacotherapy-responsive CFTR mutation identified, an evolution of the drug approval process now permits drug label expansion based on in vitro physiological response testing. Indeed, the development of novel patient cell-based methods such as cultured intestinal organoids [3, 4] or nasal epithelial spheroids [5] permit in vitro testing of targeted CFTR molecular therapies. Here, we report the use of a novel preclinical test in one such patient to demonstrate patient-specific functional correction of CFTR ex vivo followed by initiation of therapy and functional correction in vivo. Further, while adverse respiratory effects have been reported with lumacaftor/ivacaftor therapy [6], a comprehensive description of the treatment adjustment period has not been previously reported, here we provide a detailed account of this phenomenon.

A 38-year-old man with CF diagnosed by sweat testing with a typical clinical course and inconclusive CFTR genotyping became increasingly concerned about his health due to a declining lung function despite strong compliance with self-administered CF therapies. Inspired by the emergence of CFTR mutation-specific pharmacotherapy and the availability of improved genetic testing, this extremely motivated patient sought CFTR gene sequencing analysis, which identified a rare, poorly characterised mutation Ser1159Pro (c.3475T $>$ C) [7] and the common class II mutation Phe508del (c.1521_1523delCTT). He conducted his own personal investigation among the CF patient community and then initiated the further consideration of evaluation and treatment options within his own care team. He underwent preclinical CFTR therapeutic response testing [5] in which his nasal epithelial cells were isolated, cultured as spheroids and air-liquid interface (ALI) monolayers, and responses to ivacaftor and/or lumacaftor were measured.

This protocol was approved by the Cincinnati Children's Hospital Medical Center Institutional Review Board. Nasal cell culture and analysis was performed as previously reported [5, 8].

Nasal epithelial cells were obtained from both inferior turbinates by curettage, pooled, dispersed with Accutase (Innovative Cell Technologies Inc., San Diego, CA, USA), suspended in DMEM-F12-based media containing antibiotics and rho-kinase inhibitor, and seeded into dishes coated with VitroCol (Advanced BioMatrix, San Diego, CA, USA), pre-seeded with irradiated mouse embryonic fibroblast feeder cells. Media was changed daily, antibiotics were removed after 5 days, and cultures maintained until nasal cells reached confluence. Cells were dissociated with $0.1 \%$ trypsin and then maintained as spheroid or ALI cell monolayer cultures.

@ERSpublications

Pharmacotherapeutic response testing identified CFTR Ser1159Pro as responsive to cystic fibrosis modulator therapy http://ow.ly/dvsq30jcBMw

Cite this article as: McCarthy C, Brewington JJ, Harkness B, et al. Personalised CFTR pharmacotherapeutic response testing and therapy of cystic fibrosis. Eur Respir J 2018; 51: 1702457 [https://doi.org/10.1183/13993003.02457-2017]. 
Nasal epithelial cell spheroid culture and measurement of cAMP-induced swelling: cultured nasal cells were suspended in growth factor-reduced matrigel at 500000 cells $\cdot \mathrm{mL}^{-1}$ and $100-\mu \mathrm{L}$ aliquots were seeded in $16-\mathrm{mm}$ four-well plates. After incubation $\left(37^{\circ} \mathrm{C}, 5 \% \mathrm{CO}_{2}, 30 \mathrm{~min}\right)$, DMEM-F12-based media was added to each well and then changed daily during culture until the spheroids were mature (7-10 days). Nasal epithelial cell spheroid swelling was measured by time-lapse photomicroscopy in an incubated microscope chamber by capturing bright field images before (baseline) and $1 \mathrm{~h}$ after addition of phosphate buffered saline containing forskolin $(10 \mu \mathrm{M})$ and $\operatorname{IBMX}(100 \mu \mathrm{M})$ to the media to increase intracellular cAMP levels. Some wells were pretreated for $48 \mathrm{~h}$ with lumacaftor $(3 \mu \mathrm{M})$ and/or some received ivacaftor $(1 \mu \mathrm{M})$ at the time of stimulation. Nasal epithelial cell spheroid swelling was measured as the change in luminal area of a spheroid before and after stimulation determined from paired photomicrographs by outlining the spheroid lumen manually followed by automated measurement of the luminal area.

ALI cell monolayer culture and measurement of cAMP-induced short circuit current: cultured nasal cells were suspended in DMEM-F12-based media and seeded onto Transwell plates (Corning, Inc., NY, USA) clear permeable inserts $(0.4 \mu \mathrm{m}$ pore size $)$ coated with type-IV collagen at approximately $260000 \mathrm{cells} \cdot \mathrm{cm}^{-2}$. Cells were maintained until maturity (4-6 weeks), until transepithelial resistance reached approximately $500-1000 \mu \Omega \cdot \mathrm{cm}^{-2}$. Select inserts were pretreated with lumacaftor $(3 \mu \mathrm{M})$ for $48 \mathrm{~h}$ prior to study. Inserts were mounted in Ussing chambers and studied in an asymmetric chloride solution to produce a basolateral-to-apical $\mathrm{Cl}^{-}$secretory gradient. Cells were treated with $100 \mu \mathrm{M}$ amiloride to block ENaC and sodium transport. Forskolin $(10 \mu \mathrm{M})$ and IBMX $(100 \mu \mathrm{M})$ were then added in both compartments to increase cAMP and stimulate CFTR. For select inserts, ivacaftor $(1 \mu \mathrm{M})$ was added to potentiate CFTR. Finally, CFTR Inhibitor-172 $(10 \mu \mathrm{M})$ was added to the apical compartment to block CFTR currents. Short-circuit current and resistance were measured under voltage-clamp conditions.

Pharmacotherapeutic response testing demonstrated that nasal epithelial spheroids treated with lumacaftor/ ivacaftor swelled in response to cAMP-stimulation, with a mean increase in luminal area of $22.27 \%$ compared with $9.37 \%$ in untreated spheroids $(p=0.002)$. Correction was also noted in response to ivacaftor alone $(p=0.005)$ and a trend towards correction was noted with lumacaftor alone $(p=0.065)$. These results indicate a pharmacological correction of Ser1159Pro/Phe508del nasal epithelial spheroids to lumacaftor/ ivacaftor ex vivo (figure 1a and b), which is important before initiating expensive, lifelong therapy.

Confirmation of this pharmacological response was demonstrated by treatment of ALI monolayers with lumacaftor and ivacaftor alone or in combination. All treatment conditions resulted in an increased short-circuit current confirming the functional correction of CFTR. (figure 1c) and the correlation between individual ALI and spheroid responses has previously been reported [5].

Having first demonstrated pharmacodynamic correction in vitro, the effects of lumacaftor/ivacaftor therapy were confirmed in vivo by sweat testing 5 weeks after initiating therapy. The patient's sweat chloride test normalised to $11 \mathrm{mEq} \cdot \mathrm{L}^{-1}$ in both arms from abnormal values of $68 \mathrm{mEq} \cdot \mathrm{L}^{-1}$ and $54 \mathrm{mEq} \cdot \mathrm{L}^{-1}$ recorded 2 weeks prior to initiation of therapy (figure 1d).

1 day after initiation of ivacaftor/lumacaftor therapy the patient noted dyspnoea at rest, slight wheezing, chest discomfort and a drop in forced expiratory volume in $1 \mathrm{~s}$ (FEV1) to 50\% predicted. Over the next 3 days he reported worsening dyspnoea, continued wheezing and chest pain, and the onset of systemic "flu-like" symptoms including malaise, generalised muscle aches, headache, lethargy and loss of appetite. There was a persistent reduction in FEV1 of $49-50 \%$ predicted. After 5 days of therapy he noted an improvement in pulmonary and systemic symptoms despite persistent chest tightness and the onset of drenching night sweats. Following 1 week of treatment the patient reported resolution of systemic symptoms and continued improvement in chest tightness, dyspnoea, wheezing, but an increase in cough and sputum production (2-3 tablespoons per day). FEV1 increased to values ranging between $66 \%$ and $77 \%$ predicted. During weeks three and four of therapy, the patient reported minimal dyspnoea, decreased wheeze, minimal chest tightness, and production of thin, clear to slightly yellow sputum. He resumed vigorous exercise, which resulted in transient chest tightness, significant wheezing, increased sputum production and post-exercise streaking haemoptysis, all of which resolved within hours after discontinuing exercise.

Approximately 1 month after starting lumacaftor/ivacaftor therapy, the patient reported "feeling that his lungs were clear". His previous baseline spontaneous cough was absent, with cough only occurring during airway clearance therapy, productive of a tablespoon of non-viscous yellow-white sputum and incidentally the patient also noted a resolution of aquagenic wrinkling, a reported effect of ivacaftor therapy [9]. After 46 days of therapy the $\mathrm{FEV} 1$ had increased to $79 \%$ predicted; representing an $11 \%$ improvement upon the pre-treatment baseline of $68 \%$ predicted (figure 1e).

This report identified Ser1159Pro as a CFTR allele amenable to ivacaftor therapy by use of a novel CFTR-treatment response test performed with patient-derived nasal epithelial spheroids. Results support 
a)
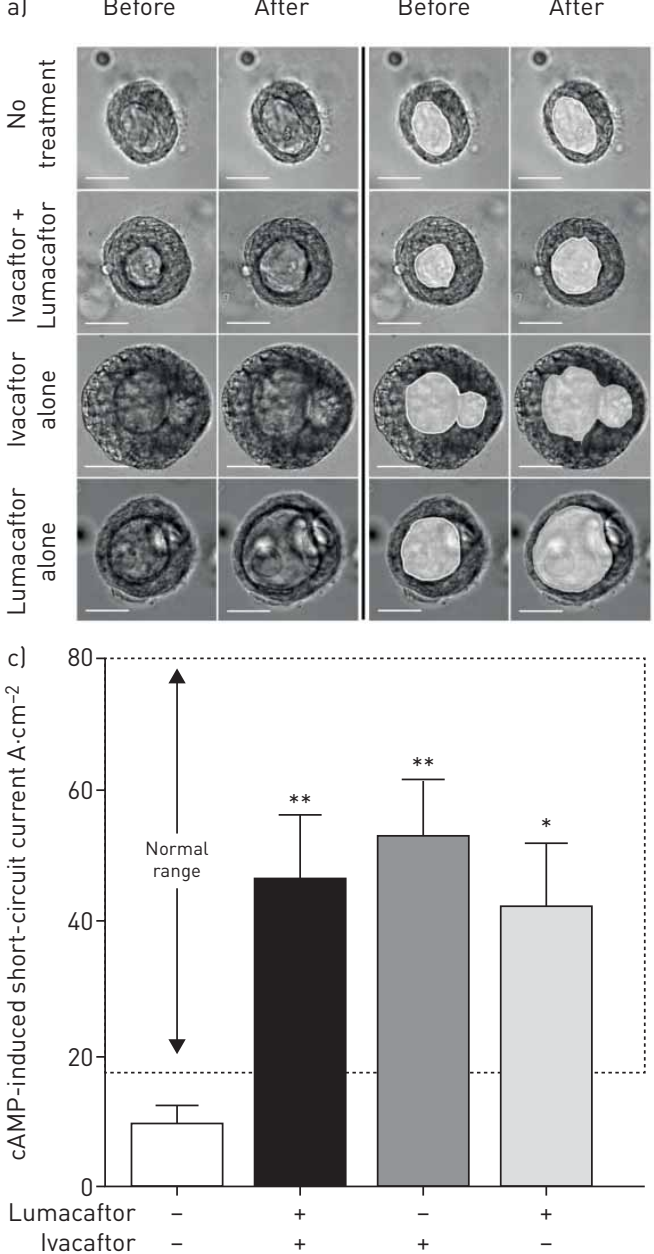
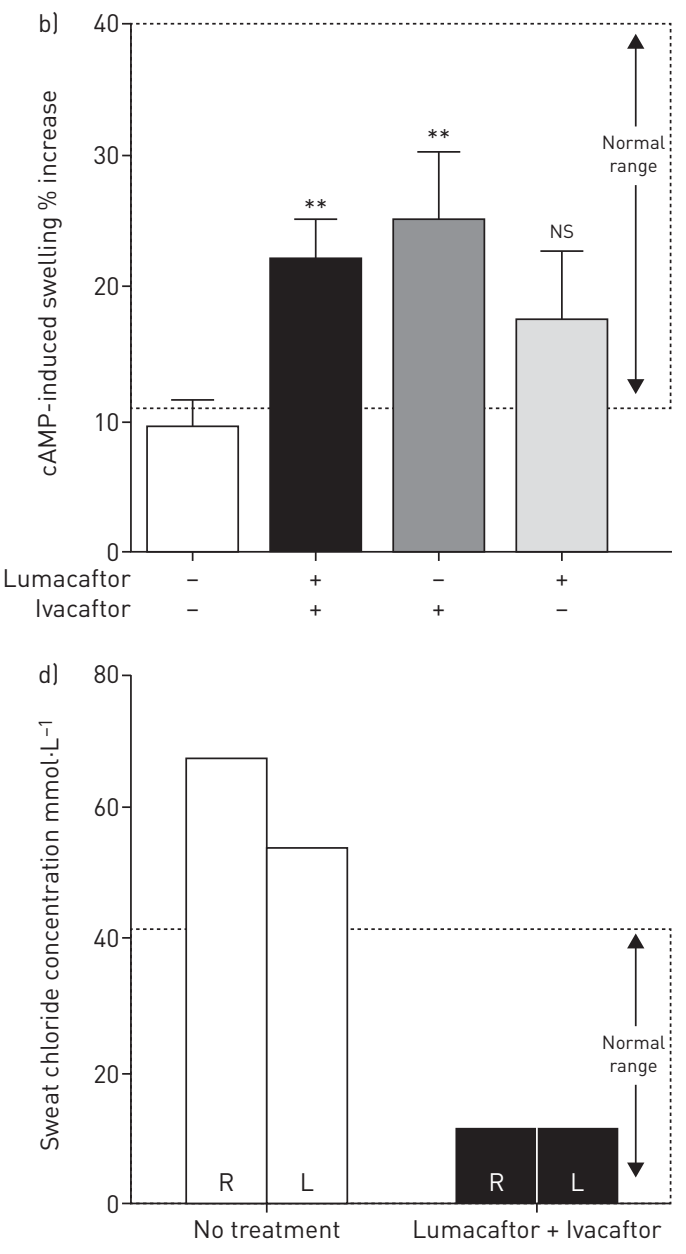

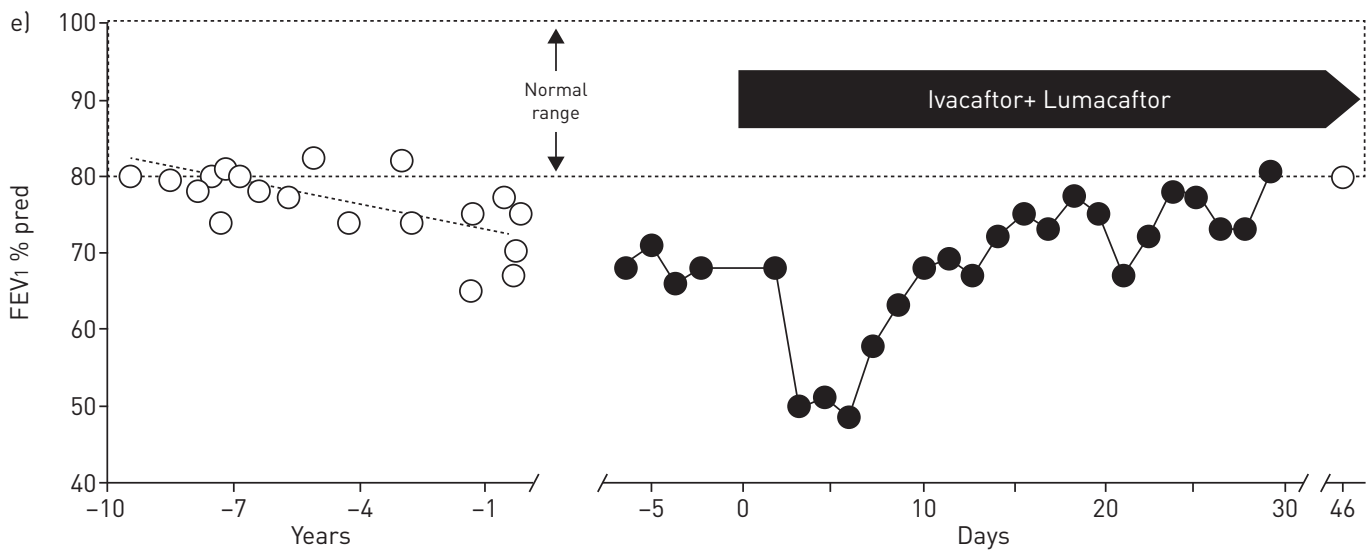

FIGURE 1 Preclinical testing and clinical responses to cystic fibrosis transmembrane conductance regulator (CFTR)-specific pharmacotherapy. Nasal epithelial cells were obtained from the patient, maintained as spheroid or air-liquid interface (ALI) cell monolayer cultures, and used to measure cAMP-induced, CFTR-mediated physiological responses ex vivo (a-c). Oral lumacaftor/ivacaftor therapy was then initiated and treatment-related biomarker and clinical outcome responses were measured (d-e). a) Representative photomicrographs of nasal cell spheroids before and after addition of forskolin and IBMX to stimulate cAMP-induced activation of CFTR-dependent swelling in the absence or presence of ivacaftor plus lumacaftor treatment. White masking indicates the spheroid lumen. Scale bar: $100 \mu \mathrm{M}$. b) Change in cAMP-induced spheroid luminal

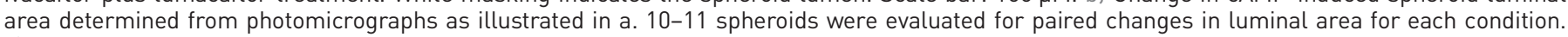
c) Change in CAMP-induced short-circuit current for ALI cell monolayers cultured with or without lumacaftor and/or ivacaftor pre-treatment as indicated. Three to four ALI cell monolayers were evaluated for each condition. d) Standard clinical sweat chloride test results obtained 2 weeks before and 7 weeks after lumacaftor/ivacaftor, demonstrating treatment-related normalisation of sweat chloride in right (R) and left ( $\mathrm{L}$ ) arms.

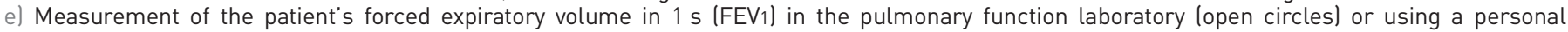

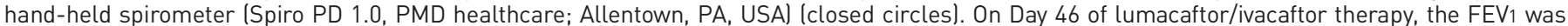
confirmed at $79 \%$ of the predicted value determined in the pulmonary function laboratory. T-bars indicate the standard error. ${ }^{* *}$ : $p<0.01 ;{ }^{*}: p<0.05$;

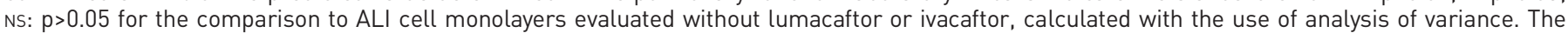
normal range of values shown in b and c represent 95\% confidence intervals in healthy subjects as previously reported [4]. 
expanding the list of CFTR mutations appropriate for CFTR-specific pharmacotherapy to include Ser1159Pro and the feasibility of the evolving approval paradigm based on in vitro cell-based CFTR functional testing. While both nasal and intestinal cells appear suitable for this purpose, nasal epithelial cells bear closer resemblance to lower respiratory epithelium than to intestinal epithelium; and in our experience, patients found the nasal epithelial cell approach simple and tolerable. Finally, this patient's exceptional perseverance resulted in comprehensive description of the initial adjustment period that can occur in response to initiation of lumacaftor/ivacaftor therapy as well as the recovery and positive clinical response that follows it, which may be of use to $\mathrm{CF}$ patient and physician communities.

Cormac McCarthy $\circledast^{1,2,3}$, John J. Brewington ${ }^{2}$, Beth Harkness ${ }^{4}$, John P. Clancy ${ }^{2}$ and Bruce C. Trapnell ${ }^{1,2,3}$

${ }^{1}$ Translational Pulmonary Science Center, Division of Pulmonary Biology, Children's Hospital Medical Center, Cincinnati, OH, USA. ${ }^{2}$ Division of Pulmonary Medicine, Children's Hospital Medical Center, Cincinnati, OH, USA. ${ }^{3}$ Division of Pulmonary, Critical Care, and Sleep Medicine, University of Cincinnati College of Medicine, Cincinnati, OH, USA. ${ }^{4}$ Children’s National Medical Center, Washington, DC, USA.

Correspondence: Bruce C. Trapnell, Division of Pulmonary Biology, Cincinnati Children's Hospital Medical Center, 3333 Burnet Avenue, Cincinnati, OH 45229-3039, USA. Email: Bruce.Trapnell@cchmc.org

Received: Oct 052017 | Accepted after revision: March 122018

Conflict of interest: None declared.

\section{References}

1 Ramsey BW, Davies J, McElvaney NG, et al. A CFTR potentiator in patients with cystic fibrosis and the G551D mutation. N Engl J Med 2011; 365: 1663-1672.

2 Wainwright CE, Elborn JS, Ramsey BW, et al. Lumacaftor-ivacaftor in patients with cystic fibrosis homozygous for Phe508del CFTR. N Engl J Med 2015; 373: 220-231.

3 Dekkers JF, Berkers G, Kruisselbrink E, et al. Characterizing responses to CFTR-modulating drugs using rectal organoids derived from subjects with cystic fibrosis. Sci Transl Med 2016: 8: 344ra384.

4 Dekkers JF, Gogorza Gondra RA, Kruisselbrink E, et al. Optimal correction of distinct CFTR folding mutants in rectal cystic fibrosis organoids. Eur Respir J 2016; 48: 451-458.

5 Brewington JJ, Filbrandt ET, LaRosa FJ III, et al. Detection of CFTR function and modulation in primary human nasal cell spheroids. J Cyst Fibros 2018: 17: 26-33.

6 Hubert D, Chiron R, Camara B, et al. Real-life initiation of lumacaftor/ivacaftor combination in adults with cystic fibrosis homozygous for the Phe508del CFTR mutation and severe lung disease. J Cyst Fibros 2017; 16: 388-391.

7 Simakova T, Kondratyeva E, Avakian L, et al. Identification of a novel, CF-causing compound genotype (p.S1159P and p.Y569H) using an NGS-based assay: novel CF-causing compound p.S1159P and p.Y569H genotype. Gene 2016; 575: 567-569.

8 Rowe SM, Pyle LC, Jurkevante A, et al. DeltaF508 CFTR processing correction and activity in polarized airway and non-airway cell monolayers. Pulm Pharmacol Ther 2010; 23: 268-278.

9 Grasemann H, Ratjen F, Solomon M. Aquagenic wrinkling of the palms in a patient with cystic fibrosis. N Engl J Med 2013; 369: 2362-2363. 